\title{
Design and in Silico Construction of New Efficient Antibodies Against Gastric Tumor-Associated Antigens Towards Introducing Combined Strategy for Vaccine Tumor Therapy
}

\author{
Ghazaleh Ghavami ${ }^{1}$, Sakineh Shahidi ${ }^{1}$, Zohreh Mazinani $^{1}$, Sholeh Maslehat ${ }^{1}$, Soroush Sardari ${ }^{*}$
}

1 Drug Design and Bioinformatics Unit, Department of Medical Biotechnology, Biotechnology Research Center, Pasteur Institute of Iran, Tehran, Iran.

Accepted Mar 11, 2015

\begin{abstract}
Introduction: Gastric cancer, as the second reason of cancer mortality around the world, is defined as one of the serious health problems and a wide range of current research is focused to overcome the challenges relevant to its successful therapy. Employing recent approaches in the field of vaccine tumor therapy and immunotherapic strategies such as utilization of monoclonal antibodies can play significant roles in achieving efficient therapeutic means to combat the disease. Methods: In this study, computational methods including three-dimensional modeling, based on sequence combinational pattern identification together with docking procedures have been employed for design and in silico construction of prolific and effective antibody structures against HER-2 and MAGE-3 antigens. Results: These methods led to introduction of novel and efficient anti-HER2 and anti-MAGE3 antibodies. For instance, combinational pattern of bH1 antibody (light chain) and IGG1-KAPPA 4D5 antibody (heavy chain) which obtained an exceptional score of $844.83 \mathrm{KJ} / \mathrm{mol}$ as the new candidate for cancer vaccine therapy, whereas Herceptin scored $453.4 \mathrm{KJ} / \mathrm{mol}$. In addition, the suggested pattern for designing antibody against MAGE-3 with combination of bH1 antibody (heavy chain) and HLA-A 0201 (light chain) achieved acceptable score of $640.8 \mathrm{KJ} / \mathrm{mol}$. Conclusion: It is envisaged that the results obtained from the current research could be utilized to develop efficient combined strategies for vaccine therapy of gastric cancer.
\end{abstract}

KEYWORDS: Gastric cancer, Vaccine therapy, Antibody, HER-2, MAGE-3, in silico, Docking

\section{INTRODUCTION}

Cancer with more than 11 million deaths per year is the second principal cause of death. It is estimated that there will be 16 million new cases every year by 2020. Deaths from cancer are predicted to be rising, with an expected 9 million people dying from cancer in 2015 and 11.4 million dying in 2030, worldwide. Additionally, gastric cancer is the second most common form of cancer-related deaths, globally. Cancer immunotherapy has been introduced as an influential and promising clinical trend for the treatment of cancer and it has been successful in treatment of breast and prostate cancers as well as melanoma [1].

Tumors are appeared by the progressive growth and proliferation of non-programmed cells. It is probable that cancerous cells occur daily in healthy individuals; however in the majority of cases, they are detached by the immune system

*Corresponding Author: Soroush Sardari, Ph.D; Drug Design and Bioinformatics Unit, Department of Medical Biotechnology, Biotechnology Research Center, Pasteur Institute of Iran, Tehran, Iran. No. 69, Pasteur Ave, Tehran, Iran, 1316943551.

Email: sardari@pasteur.ac.ir, ssardari@hotmail.com.

Tel/Fax: (+98) 2166954324 and cannot grow into malignancies. The above-mentioned capability of the immune system to sense and eliminate cancerous cells is known as "immune surveillance". Cancerous cells are detected by innate and adaptive immune cells which sense the local environment of a malignancy when it begins to remodel the stroma [1]. The crucial aim of immunotherapy is overcoming malignancies by inducing an efficient immune response against the cancerous cells. In theory, if particular antigens could be recognized in the forerunner lesions leading to a tumor, the immune responses can be used to eradicate them which may prevent the cancerous cells to convert into a malignancy [2]. Gastric cancer is potentially a suitable target for such defensive immunotherapy as it progresses over an extended period of time and its premalignant lesions are clearly identifiable which if effectively targeted, their development to forthright tumor could be prevented. Current immune therapies of gastric tumors are frequently based on utilizing monoclonal antibodies (vaccine and drugs), cytotoxic immunocytes, or gene transferred vaccines [3]. Experimental vaccination methodologies involve using whole protein and peptide vaccines, based on recognition of certain peptides by cytotoxic 
$\mathrm{T}$ lymphocytes and helper $\mathrm{T}$ lymphocytes. Tumor rejection antigens such as melanoma-associated antigen 3 (MAGE-3) [4] and HER-2/neu [5] are two instances of antigens detectable by cytotoxic $\mathrm{T}$ cells which can be selectively expressed in human tumors, including gastric cancer. Monoclonal antibodies against the above antigens can play a role in shaping the cellular immune system toward destroying the tumors. The detection and neutralization of cancerous antigens by the antibodies are initiated based on binding of such antibodies to the antigens, often to the surface proteins, through specific atomic interactions between the antibody and an epitope, as a specified region of the antigen. The designing and synthesizing of a new generation of monoclonal antibodies or the rationale for optimizing the current ones in order to accelerate vaccine development is based on discovering the best points of antigenantibody interaction; due to the fact that most current vaccines are based on the generation of neutralizing antibody responses [6].

Current preclinical and clinical researches have introduced the rationale for combining vaccine therapy with conventional therapies for patients with developed cancers. In fact, anticancer vaccines in the frame of monotherapy for the tumors in advanced stages have shown minimal clinical efficacy. On the subject of combined immunotherapy strategy, Hodi et al. [7] have published a Phase I clinical investigation in which they have used anti-CTLA-4 as a therapeutic modality. Their result has indicated that the prior immune memory responses may be amplified by using an anti-CTLA-4 antibody (i.e. Ipilimumab; Medarex, Princeton, NJ). This antibody combined with a peptide vaccine against melanoma could alter the level and/or avidity of the antigen-specific T cells. Furthermore, the preliminary findings have shown that a whole tumor-cell vaccine combined with ipilimumab resulted in a high percentage of patients with declines in PSA when compared to either treatment modality alone [7].

As the main phase of monoclonal antibody designing, the best way to study the atomic interactions is to obtain the threedimensional structure of the antibody-antigen complexes. Conventionally, this is carried out by experimental techniques like X-ray crystallography, an often long and laborious procedure with a wide range of failure rate [6]. Hence, the advanced algorithms and processing tools based on computational techniques have been utilized in silico for the structural characterization of intermolecular complexes. Computational modeling of the three-dimensional structures and docking can supply a rapid and inexpensive route to introduce the best monoclonal antibody-based vaccines for the experimental determinations and to reject the unsuitable candidates. As the first step of the current investigation, the in silico modeling was employed to design novel antibodies against HER-2 and MAGE-3 antigens based on combinational patterns. During the next steps, computational docking was utilized to investigate the interactions of the designed antibodies with antigens toward introducing a novel and efficient strategy for vaccine tumor therapy or optimizing the existing ones for successful gastric cancer immunotherapy.

\section{MATERIALS and METHODS}

\section{Combinational patterning of antibodies}

The current research was constructed based on employing variants of existing antibodies against HER-2 antigen [8] with the greatest potency, in order to design and introduce new antibody patterns with targeted qualified activity based on combinational methods. On the subject of mentioned strategy; at the first step, the Fab fragments related to I. bH1 antibody (A1) [9], II. IGG1-KAPPA 4D5 antibody (A2) [10], III. MOR09825 antibody (A3) [11] and IV. Fab37 engineered antibody (A4) [12] were selected and their FASTA sequences were obtained from http://www.ncbi.nlm.nih.gov/protein/. During the next step, the possible combinations of their heavy and light chains involving Fab fragment were created towards novel antibody modeling. Additionally, the heavy chain of Fab fragment related to bH1 antibody and the sequence of Human Class I Histocompatibility Antigen (HLA-A 0201) were utilized to design an efficient antibody against MAGE-3.

Antibody modeling against HER-2 and MAGE-3

PIGS (Prediction of ImmunoGlobulin Structure) as a webbased server (http://circe.med.uniroma1.it/pigs/) was utilized for the automatic prediction of antibody structure in the frame of canonical structure method [13]. In this method, the sequence of each variable domain ( $\mathrm{VL}$ and $\mathrm{VH}$ ) of the new antibody (target) was independently aligned with the analogous variable domain sequences of all the immunoglobulins of defined structure by standard database searching (i.e. BLAST) and multiple sequence alignment (i.e. Clustalw). In the next step, the backbone structure of the framework was modeled based on the known structures with highest sequence identity as templates. In the same way, the conformation of the CDR loops was predicted in the frame of identified templates with the identical canonical loop conformation and high sequence identity. Lastly, the model was refined based on only some cycles of energy minimization to improve the stereochemistry. The generated sequences involving light and heavy chains of the patterned antibodies were then used as inputs of PIGS for computational modeling procedure.

\section{In silico evaluation of the constructed antibodies}

After generating the starting structures of new antibodies based on computational modeling, they were brought together with certain antigens of HER-2 and MAGE-3 (their threedimensional structures were obtained from RCSB Protein Data Bank) by the computational docking. During the docking process, the scoring function rewarded the positive interactions between the receptor (antibody) and the ligand (antigen) while penalized the negative interactions. Assuming that the suitable and accurate biological complexes have the lowest energy, the calculation of energy levels can then be used to predict the future biological activity and potency of a biomolecule [14]. Consequently, the docking algorithm was used to calculate the energy levels within the antibody-antigen complex at interacting points, based on electrostatic and shape parameters via different statuses of their interactions. The calculating and scoring functions were carried out by accounting for the biophysical considerations such as hydrophobic and electrostatic interactions, salt bridges, hydrogen bonds, etc., in addition to statistical and empirical parameters like the degree of conserved residues at the interface. In this research, to predict the potency of the designed antibodies against certain antigens, docked conformations and interaction energies were obtained utilizing HEX 6 (2008). All programs were run on an IBM compatible computer running at $8 \mathrm{~GB}$ RAM and $3.4 \mathrm{GHz}$ Quadruple Xeon CPU.

\section{RESULTS}

Combinational designing and modeling of the novel antibodies 
As mentioned previously, to design novel efficient antibodies against two of antigens involved in gastric cancer, namely HER-2 and MAGE-3, the sequences of Fab fragments in relation to the known enhanced variants and engineered antibodies (A1-A4) as well as bH1 antibody plus HLA-A 0201 (Fig. 1) were used to pattern 13 new combinational sequences (Table 1).

Furthermore, PIGS web-based server was used for antibody modeling, based on light and heavy chains of the 13 designed antibodies as test groups. Additionally, the FASTA sequences of exact antibodies (A1-A4) as well as Herceptin (the therapeutic monoclonal antibody that binds HER2) were utilized to model by PIGS as a control group. As a result, the three-dimensional structures for 18 antibodies including the test and the control groups were modeled, optimized and generated for the docking procedure.

Computational evaluating of the designed antibodies against HER-2 and MAGE-3

The in silico evaluation of the newly designed antibodies were performed based on computational docking procedure of antibody-antigen complexes by HEX 6 software in the frame of calculating shapes and electrostatic parameters. Moreover, the same process was carried out for the control group. The entire resulted scores of the energy calculations were generated and sorted (Table 2) to introduce novel and efficient antiHER2 and anti-MAGE3 antibodies. The results showed that one of the designed antibodies (i.e Abl) consisting of combinational pattern of bH1 antibody (light chain) and IGG1KAPPA 4D5 antibody (heavy chain) obtained an exceptional score of $844.83 \mathrm{KJ} / \mathrm{mol}$ as the new candidate for cancer vaccine therapy in comparison with Herceptin which scored $453.4 \mathrm{KJ} / \mathrm{mol}$ (Fig. 2).

In addition, the suggested pattern for designing antibody against MAGE-3 with combination of bH1 antibody (heavy chain) and HLA-A 0201 (light chain) achieved an acceptable score of $640.8 \mathrm{KJ} / \mathrm{mol}$ which can be considered promising for developing efficient candidates for targeting MAGE-3 during cancer immunotherapy in future.

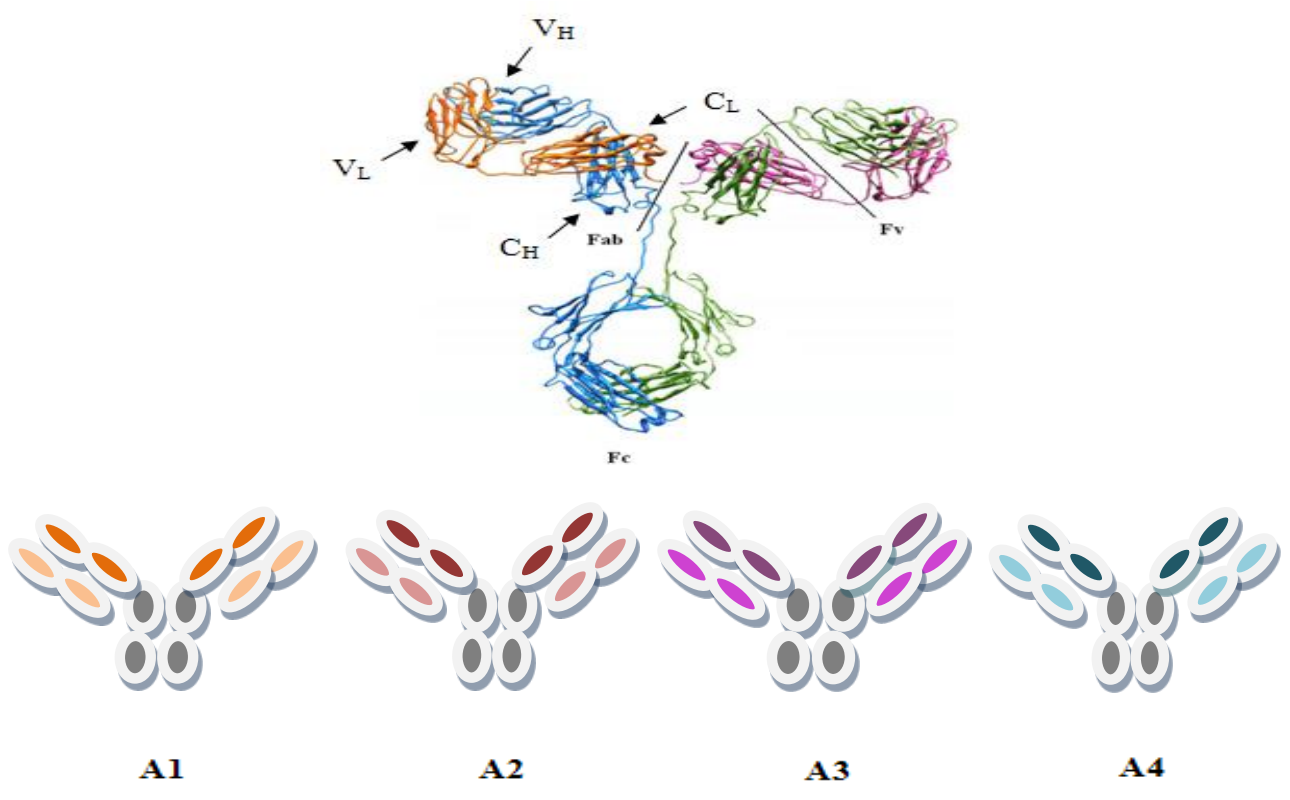

Fig. 1. The Fab fragments related to I. bH1 antibody (A1), II. IGG1-KAPPA 4D5 antibody (A2), III. MOR09825 antibody (A3) and IV. Fab37 engineered antibody (A4) were used for combinational patterning of the antibodies.
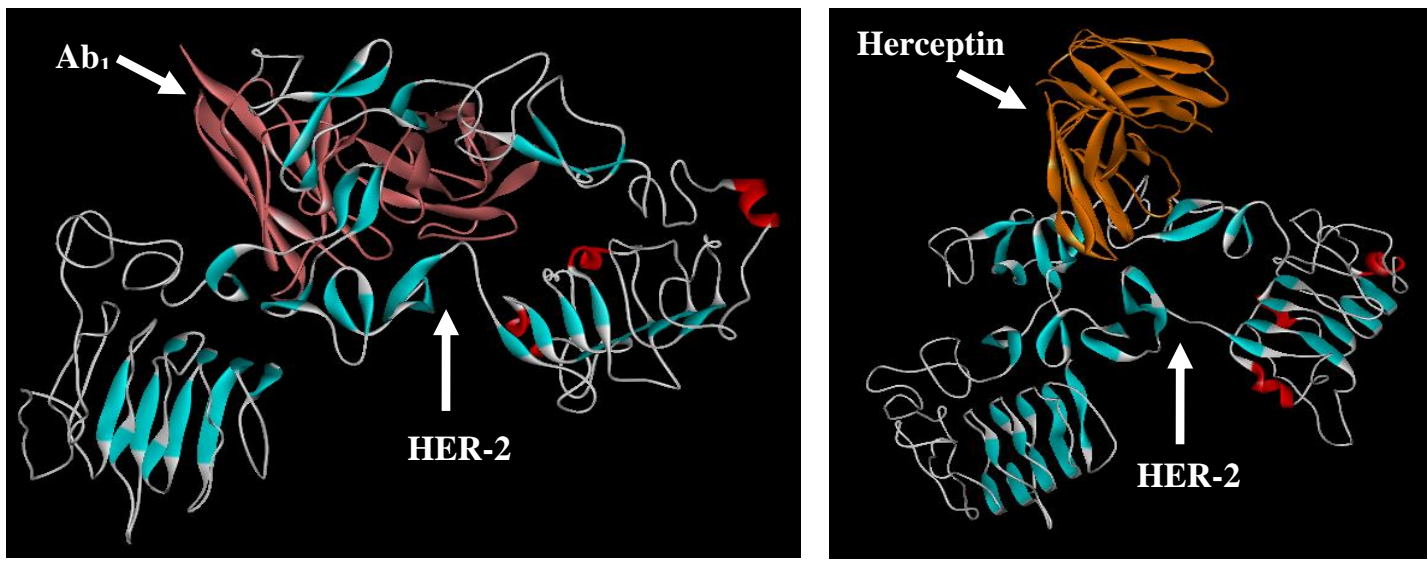

Fig. 2. The three-dimensional structures of docked antibody-antigen complexes, the newly-designed antibody Abl consisting of combinational pattern of bH1 antibody (light chain) and IGG1-KAPPA 4D5 antibody (heavy chain) could achieve an exceptional score in comparison with Herceptin. 
Table. 1. The pattern of new 13 combinational sequences of antibodies.

\begin{tabular}{|c|c|c|c|c|c|c|c|c|c|}
\hline $\begin{array}{l}\text { Designed } \\
\text { antibody }\end{array}$ & $(3)$ & $(3$ & 0 & $(3)$ & 0 & 0 & 0 & 3 & $\begin{array}{l}\text { HLA-A } \\
0201\end{array}$ \\
\hline $\mathbf{A b} \mathbf{b}_{\mathbf{a}}$ & & & & & $\checkmark$ & & & $\checkmark$ & \\
\hline $\mathbf{A} \mathbf{b}_{\mathbf{b}}$ & & $\checkmark$ & & & $\checkmark$ & & & & \\
\hline $\mathbf{A} \mathbf{b}_{\mathbf{c}}$ & & & & $\checkmark$ & $\checkmark$ & & & & \\
\hline $\mathbf{A} \mathbf{b}_{\mathrm{d}}$ & & & & & & $\checkmark$ & $\checkmark$ & & \\
\hline $\mathbf{A b} \mathbf{b}_{\mathbf{e}}$ & & $\checkmark$ & & & & & $\checkmark$ & & \\
\hline $\mathbf{A} \mathbf{b}_{\mathbf{f}}$ & & & & $\checkmark$ & & & $\checkmark$ & & \\
\hline $\mathbf{A b} \mathbf{b}_{\mathrm{g}}$ & $\checkmark$ & & & & & $\checkmark$ & & & \\
\hline $\mathbf{A} \mathbf{b}_{\mathbf{h}}$ & $\checkmark$ & & & & & & & $\checkmark$ & \\
\hline $\mathbf{A} \mathbf{b}_{\mathbf{i}}$ & $\checkmark$ & & & $\checkmark$ & & & & & \\
\hline $\mathbf{A} \mathbf{b}_{\mathbf{j}}$ & & & $\checkmark$ & & & $\checkmark$ & & & \\
\hline $\mathbf{A} \mathbf{b}_{k}$ & & & $\checkmark$ & & & & & $\checkmark$ & \\
\hline $\mathbf{A b} \mathbf{b}_{1}$ & & $\checkmark$ & $\checkmark$ & & & & & & \\
\hline $\mathbf{A} \mathbf{b}_{\mathbf{m}}$ & $\checkmark$ & & & & & & & & $\checkmark$ \\
\hline A1 & $\checkmark$ & $\checkmark$ & & & & & & & \\
\hline A2 & & & $\checkmark$ & $\checkmark$ & & & & & \\
\hline A3 & & & & & $\checkmark$ & $\checkmark$ & & & \\
\hline A4 & & & & & & & $\checkmark$ & $\checkmark$ & \\
\hline
\end{tabular}

Table. 2. Results of computational docking between antibodies from test and control groups with certain antigens.

\begin{tabular}{|l|l|l|}
\hline Antibody & Antigen & $\begin{array}{l}\text { Score } \\
(\mathbf{K J} / \mathbf{m o l})\end{array}$ \\
\hline $\mathrm{Ab} \mathrm{a}_{\mathrm{a}}$ & HER-2 & -481.0 \\
\hline $\mathrm{A} \mathrm{b}_{\mathrm{b}}$ & HER-2 & -822.6 \\
\hline $\mathrm{A} \mathrm{b}_{\mathrm{c}}$ & HER-2 & -380.3 \\
\hline $\mathrm{A} \mathrm{b}_{\mathrm{d}}$ & HER-2 & -777.4 \\
\hline $\mathrm{A} \mathrm{b}_{\mathrm{e}}$ & HER-2 & -795.4 \\
\hline $\mathrm{A} \mathrm{b}_{\mathrm{f}}$ & HER-2 & -396.0 \\
\hline $\mathrm{A} \mathrm{b}_{\mathrm{g}}$ & HER-2 & -784.9 \\
\hline $\mathrm{A} \mathrm{b}_{\mathrm{h}}$ & HER-2 & -464.2 \\
\hline $\mathrm{A} \mathrm{b}_{\mathrm{i}}$ & HER-2 & -404.6 \\
\hline $\mathrm{A} \mathrm{b}_{\mathrm{j}}$ & HER-2 & -787.9 \\
\hline $\mathrm{A} \mathrm{b}_{\mathrm{k}}$ & HER-2 & -336.9 \\
\hline $\mathrm{A} \mathrm{b}_{\mathrm{l}}$ & HER-2 & -844.8 \\
\hline $\mathrm{A} \mathrm{b}_{\mathrm{m}}$ & MAGE-3 & -640.8 \\
\hline $\mathrm{A} 1$ & HER-2 & -871.1 \\
\hline $\mathrm{A} 2$ & HER-2 & -414.3 \\
\hline $\mathrm{A} 3$ & HER-2 & -636.9 \\
\hline $\mathrm{A} 4$ & HER-2 & -404.1 \\
\hline $\mathrm{Herceptin}$ & HER-2 & -453.4 \\
\hline
\end{tabular}

\section{DISCUSSION}

The aim of designing and developing cancer vaccines is the activation and expansion of tumor-specific $\mathrm{T}$ cells as effector $\mathrm{T}$ cells. Indeed, the mechanism of action of therapeutic vaccines can be summarized as enhancing the pre-existing immunity, inducing a novel immunity, or leading to a more robust antitumor immune response. Tumor-associated antigens are known as antigens expressed on tumor cells with the ability to induce an immune response in the host. Both protein and peptide targets have been utilized to stimulate a specific immune response in gastric cancer [2]. Previous investigations have been performed based on peptides derived from the tumorassociated antigen HER2/neu-derived peptide and MAGE-3. Additionally, the findings resulted from vaccination using dendritic cells in combination with HER-2/neu peptide have shown considerable tumor regression in gastric cancers by using vaccine-loaded nanoparticles containing over expressing
HER-2/neu and MAGE-3 peptide/chitosan-deoxycholic acid. This method of therapy has been employed to simulate an antitumor immune response and could effectively lead to regression of tumor growth in a mouse model of gastric cancer [15]. During another investigation, the peptides derived from human vascular endothelial growth factor (VEGF) receptor 1 and vascular endothelial growth factor receptor 2 were used in combination with chemotherapy (S-1 plus cisplatin) [16] and activated a VEGF-specific cytotoxic lymphocyte response in patients with advanced gastric cancer.

As mentioned previously, the designing process of novel antibodies was included in the framework of combinational patterning, based on the sequences and functions of the known enhanced variant and engendered antibodies. One of the most potent of the known anti-HER2 is bH1 [9] that it isolated by Bostrom et al. as a variant of Herceptin on the basis of its ability to simultaneously interact with VEGF. The relevant experiments have revealed that distinct amino acids of this antibody can engage HER2 and VEGF energetically; however, there is a broad overlap between the antibody surfaces contacting the two antigens. In vitro and in vivo studies have shown that bH1 inhibits both HER2- and VEGF-mediated cell proliferation and the tumor progression in mouse models. Undoubtedly, such combined antibodies can enhance the efficiency and specification of cancer immunotherapy. Indeed, this experimental investigation has confirmed the current in silico approach using a newly designed antibody consisting of combinational pattern of bH1 antibody (light chain) and IGG1KAPPA 4D5 antibody (heavy chain) which achieved an exceptional score in comparison with Herceptin. Moreover, this new combined antibody has the specification properties, related to IGG1-KAPPA 4D5 antibody with targeting of p185HER2 (expressed in gastric adenocarcinomas at increased levels of 2/3[17]) together with compensation for inefficiency of IGG1-KAPPA 4D5 antibody for growth suppression of the HER2 antigen in this kind of tumor cells. On the subject of employing computational docking for designing proficient immunotherapy, Pedotti et al. have described diverse computational strategies for the modeling of antibodies and docking of their complexes, in order to predict the binding of two antibodies to the stalk region of influenza hemagglutinin, 
and have compared the results to identified experimental structures [6]. Additionally, Chahibi et al. have investigated an acceptable approach for targeted immunotherapy of cancer, based on designing and engineering of antibody-based drug delivery systems (ADDS) [18]. Furthermore, Burroto et al. have suggested a combined immunotherapeutic pattern [19] based on vaccine and chemotherapy methods to achieve optimal clinical benefits.

The above-mentioned studies and recent findings introduce the utilization of immunostimulatory monoclonal antibodies as vaccine adjuvants [20] for the treatment of cancer, based on boosting weak responses against immunogenic tumors or in combination with a range of other vaccines. In addition, employing cancer vaccines combined with conjugated monoclonal antibodies (standard chemotherapy drugs) [21, 22] may be a promising approach for the vaccine treatment of cancer at different stages. The current research showed that the designed novel antibodies against HER-2 and MAGE-3 can open new insights in planning efficient strategies for vaccine therapy of gastric cancer.

\section{ACKNOWLEDGEMENTS}

The authors gratefully acknowledge the Pasteur Institute of Iran for support of this study.

\section{CONFLICT OF INTEREST}

The authors declare that they have no conflict of interest.

\section{REFERENCES}

1. Matsueda S, Graham DY. Immunotherapy in gastric cancer. World journal of gastroenterology : WJG. 2014;20(7):1657-66. doi:10.3748/wjg.v20.i7.1657.

2. Burnet M. Cancer; a biological approach. I. The processes of control. British medical journal. 1957;1(5022):779-86.

3. Vollmers HP, Dammrich J, Ribbert H, Wozniak E, Muller-Hermelink HK. Apoptosis of stomach carcinoma cells induced by a human monoclonal antibody. Cancer. 1995;76(4):550-8.

4. Kono K, Rongcun Y, Charo J, Ichihara F, Celis E, Sette A et al. Identification of HER2/neu-derived peptide epitopes recognized by gastric cancer-specific cytotoxic $\mathrm{T}$ lymphocytes. International journal of cancer Journal international du cancer. 1998;78(2):202-8.

5. Appella E, Sekikawa T, Matsumoto Y, Kiessling R. Identification of HER2/neu-derived peptide epitopes recognized by gastric cancer-specific cytotoxic T lymphocytes. Int J Cancer. 1998; 78:202-8.

6. Pedotti M, Simonelli L, Livoti E, Varani L. Computational docking of antibody-antigen complexes, opportunities and pitfalls illustrated by influenza hemagglutinin. International journal of molecular sciences. 2011;12(1):226-51. doi:10.3390/ijms12010226.

7. Hodi FS, Mihm MC, Soiffer RJ, Haluska FG, Butler M, Seiden MV et al. Biologic activity of cytotoxic $\mathrm{T}$ lymphocyte-associated antigen 4 antibody blockade in previously vaccinated metastatic melanoma and ovarian carcinoma patients. Proceedings of the National Academy of
Sciences of the United States of America. 2003;100(8):4712-7. doi:10.1073/pnas.0830997100.

8. Cho HS, Mason K, Ramyar KX, Stanley AM, Gabelli SB, Denney DW, Jr. et al. Structure of the extracellular region of HER2 alone and in complex with the Herceptin Fab. Nature. 2003;421(6924):756-60. doi:10.1038/nature01392.

9. Bostrom J, Yu SF, Kan D, Appleton BA, Lee CV, Billeci K et al. Variants of the antibody herceptin that interact with HER2 and VEGF at the antigen binding site. Science. 2009;323(5921):1610-4 doi: $10.1126 /$ science. 1165480 .

10. Eigenbrot C, Randal M, Presta L, Carter P, Kossiakoff AA. X-ray structures of the antigen-binding domains from three variants of humanized anti-p185HER2 antibody 4D5 and comparison with molecular modeling. Journal of molecular biology. 1993;229(4):969-95. doi:10.1006/jmbi.1993.1099.

11. Garner AP, Bialucha CU, Sprague ER, Garrett JT, Sheng Q, Li S et al. An antibody that locks HER3 in the inactive conformation inhibits tumor growth driven by HER2 or neuregulin. Cancer research. 2013;73(19):602435. doi:10.1158/0008-5472.CAN-13-1198.

12. Fisher RD, Ultsch M, Lingel A, Schaefer G, Shao L, Birtalan S et al. Structure of the complex between HER 2 and an antibody paratope formed by side chains from tryptophan and serine. Journal of molecular biology. 2010;402(1):217-29. doi:10.1016/j.jmb.2010.07.027.

13. Marcatili P, Rosi A, Tramontano A. PIGS: automatic prediction of antibody structures. Bioinformatics. 2008;24(17):1953-4 doi:10.1093/bioinformatics/btn341.

14. Halperin I, Ma B, Wolfson H, Nussinov R. Principles of docking: An overview of search algorithms and a guide to scoring functions. Proteins. 2002;47(4):409-43. doi:10.1002/prot.10115.

15. Yang J, Li ZH, Zhou JJ, Chen RF, Cheng LZ, Zhou QB et al. Preparation and antitumor effects of nanovaccines with MAGE-3 peptides in transplanted gastric cancer in mice. Chinese journal of cancer. 2010;29(4):359-64

16. Kono K, Takahashi A, Ichihara F, Amemiya H, lizuka H, Fujii H et al Prognostic significance of adoptive immunotherapy with tumor-associated lymphocytes in patients with advanced gastric cancer: a randomized trial. Clinical cancer research : an official journal of the American Association for Cancer Research. 2002;8(6):1767-71.

17. Lewis GD, Figari I, Fendly B, Wong WL, Carter P, Gorman C et al Differential responses of human tumor cell lines to anti-p185HER2 monoclonal antibodies. Cancer immunology, immunotherapy : CII 1993;37(4):255-63.

18. Chahibi Y, Akyildiz IF, Sang Ok S. Antibody-based molecular communication for targeted drug delivery systems. Conference proceedings : Annual International Conference of the IEEE Engineering in Medicine and Biology Society IEEE Engineering in Medicine and Biology Society Annual Conference. 2014;2014:5707-10. doi:10.1109/EMBC.2014.6944923.

19. Burotto M, Singh N, Heery CR, Gulley JL, Madan RA. Exploiting synergy: immune-based combinations in the treatment of prostate cancer. Frontiers in oncology. 2014;4:351. doi:10.3389/fonc.2014.00351.

20. Gray JC, French RR, James S, Al-Shamkhani A, Johnson PW, Glennie MJ. Optimising anti-tumour CD8 T-cell responses using combinations of immunomodulatory antibodies. European journal of immunology. 2008;38(9):2499-511. doi:10.1002/eji.200838208.

21. Jiang J, Xu N, Wu C, Deng H, Lu M, Li M et al. Treatment of advanced gastric cancer by chemotherapy combined with autologous cytokine-induced killer cells. Anticancer research. 2006;26(3B):2237-42. 22. Jiang JT, Shen YP, Wu CP, Zhu YB, Wei WX, Chen LJ et al. Increasing the frequency of CIK cells adoptive immunotherapy may decrease risk of death in gastric cancer patients. World J Gastroentero. 2010;16(48):6155-62. doi:DOI 10.3748/wjg.v16.i48.6155. 